\title{
Agôn
}

Revue des arts de la scène

6 | 2013

La Reprise

\section{Métamorphoser la reprise : variations et continuité de l'Arlequin, serviteur de deux maîtres de Giorgio Strehler}

Aude Astier

\section{OpenEdition}

Journals

Édition électronique

URL : http://journals.openedition.org/agon/2736

DOI : 10.4000 /agon.2736

ISSN : 1961-8581

Éditeur

Association Agôn

Référence électronique

Aude Astier, « Métamorphoser la reprise : variations et continuité de l'Arlequin, serviteur de deux maitres de Giorgio Strehler », Agôn [En ligne], 6 | 2013, mis en ligne le 17 février 2014, consulté le 19 avril 2019. URL : http://journals.openedition.org/agon/2736 ; DOI : 10.4000/agon.2736

Ce document a été généré automatiquement le 19 avril 2019

Association Agôn et les auteurs des articles 


\title{
Métamorphoser la reprise : variations et continuité de l' Arlequin, serviteur de deux maîtres de Giorgio Strehler
}

\author{
Aude Astier
}

\section{NOTE DE L'ÉDITEUR}

Les photographies des mises en scène de l'Arlecchino servitore di due padroni convoquées dans l'article sont consultables en ligne sur le site des archives du Piccolo Teatro de Milan: http://archivio.piccoloteatro.org/eurolab/index.php?IDtitolo=29.

124 juillet 1947 : la saison inaugurale du Piccolo Teatro de Milan, fondé quelques mois auparavant ${ }^{1}$, se clôt par la création d'une pièce de Carlo Goldoni, symbole de la refondation d'un répertoire italien qui ne délaisse pas les dialectes et renoue avec un sentiment de fierté nationale, nécessaire dans cette période de reconstruction d'une nation et d'un répertoire étouffés par les années de fascisme. Rebaptisée Arlequin, serviteur de deux maîtres, pour indiquer plus clairement au public son appartenance à la Commedia dell'arte et son caractère comique, Le serviteur de deux maîtres est la première pièce de Goldoni, écrite à partir du répertoire dell'arte. Elle permet à Strehler d'aborder l'œuvre d'un auteur qu'il admire en tant que réformateur et génie de la comédie italienne, réinventeur de la Commedia dell'arte et écrivain de troupe. Le spectacle est un succès immédiat, manifestant la cohérence des partis-pris du Piccolo Teatro et la clarté des intentions de mise en scène.

23 décembre 2013: comme chaque saison ou presque depuis la mort de Strehler, la fin d'année voit la reprogrammation du spectacle, selon la régie de Giorgio Strehler et la mise en scène de Ferruccio Soleri, le second Arlequin, assisté de Stefano de Luca. 
Entre temps, la mise en scène aura connu dix éditions, dont sept signées par Giorgio Strehler et plus de 2200 représentations, cas singulier d'une mise en scène reprise et reprogrammée pendant soixante-six ans, «toujours identique et toujours différente $»^{2}$. La reprise, pour nous, sera constituée par chaque édition procédant à une variation et à une réinterprétation de la précédente et possédant un sens dépassant la simple reprogrammation commandée par le succès ou l'agencement de la saison. Elle revêt des significations et des fonctions tributaires de l'évolution de l'approche théâtrale de Strehler et du contexte historique et social qui influe sur la place accordée au théâtre. Si cette reprise s'inscrit avant tout dans la poursuite d'une recherche théâtrale liée en partie à la formation de l'acteur et consacrée à un auteur fondamental du répertoire de Strehler, elle rejoint à la mort du metteur en scène une dimension patrimoniale.

\section{Reprendre la Commedia dell'arte}

Le travail sur Le Serviteur de deux maitres de Carlo Goldoni entend réinventer une forme scénique tombée en désuétude, la Commedia dell'arte, afin de retrouver une période florissante du théâtre italien et de remettre à l'honneur le plaisir du jeu, symbole d'une énergie vitale retrouvée. Lorsque les répétitions commencent, peu de traces survivent encore de la tradition du jeu improvisé et du masque, qui s'est éteinte en Italie à la fin du XVIIIème siècle. Les acteurs et le metteur en scène, dans un premier geste d'ordre archéologique, partent à la recherche des vestiges de la Commedia dell'arte et du jeu masqué, dont ils ne savent presque rien. À partir du travail de Renato Simoni ${ }^{3}$ et de Max Reinhardt ${ }^{4}$, mais aussi des recueils de canevas qui subsistent encore et de la pièce de Goldoni, ils lancent des hypothèses et procèdent par essais. Les premières représentations de l'Arlequin se font encore avec des masques peints sur les visages, les seuls masques retrouvés faits de gaze et de cartons étant trop inconfortables et inadaptés au jeu. Les costumes sont simplement esquissés dans leurs formes et leurs motifs. La première édition se constitue comme une carcasse, une structure rudimentaire issue des premières recherches et évoquant à grands traits la Commedia dell'arte à travers la présence d'une estrade de bois de sept mètres de long et de quatre mètres de profondeur et de périactes, qui facilitent les changements de décors tout en conférant à l'espace une relative neutralité. 
Arlecchino servitore di due padroni, de Goldoni. Mise en scène de Giorgio Strehler, 1947

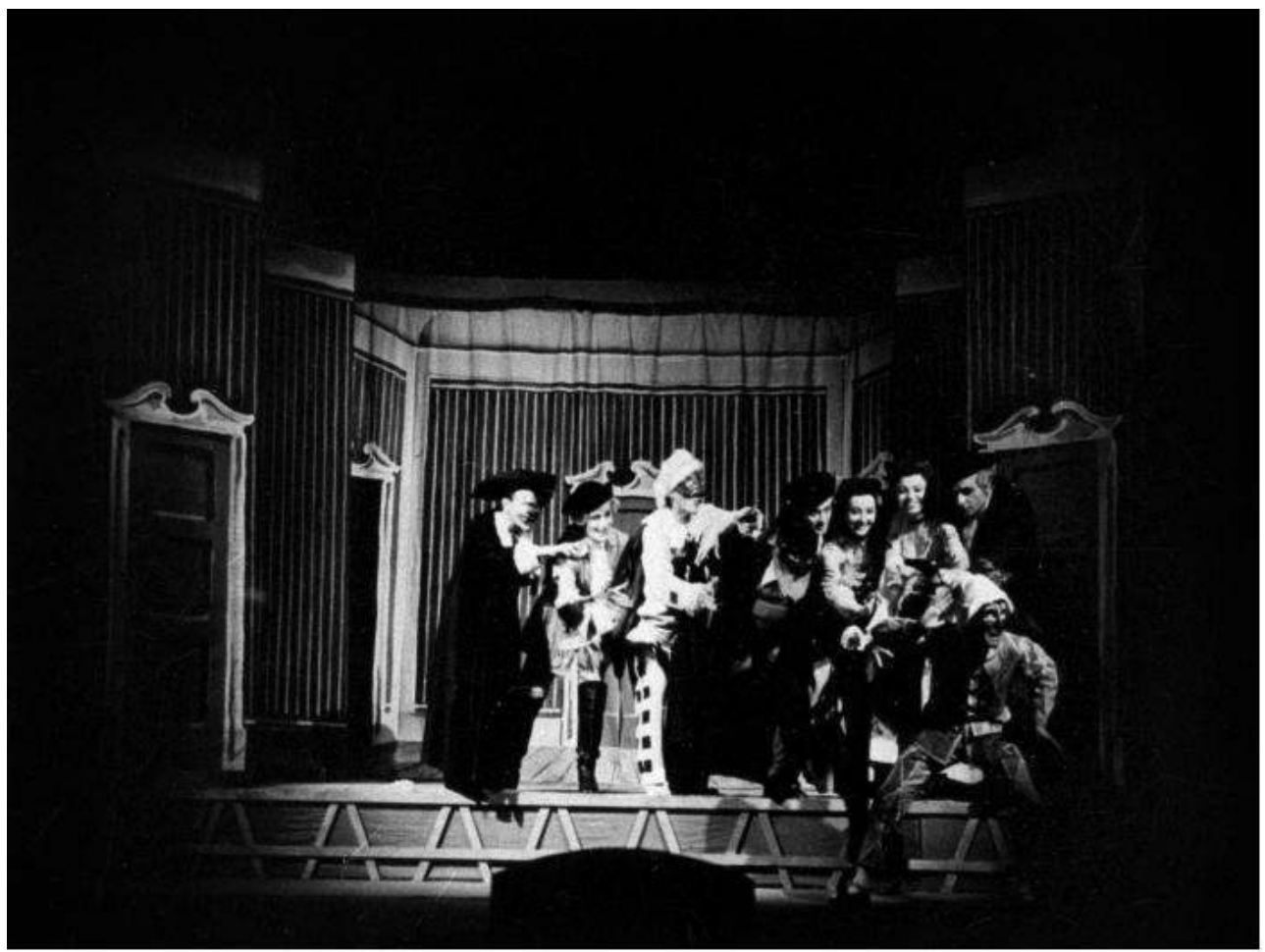

(c) Inconnu / Piccolo teatro di Milano

5 "Stylisé à la façon du théâtre de marionnettes " ${ }^{5}$, cet Arlequin réanime une des sources majeures du théâtre italien, se fondant sur les maigres traces restantes et inventant le reste, entre le fantasme d'une tradition théâtrale devenue mythique et son histoire effective. Mais déjà, la mise en scène propose l'exploration scénique des limites du possible et de la logique. Elle s'affirme comme divertissement pur, favorisant les retrouvailles avec le plaisir du jeu. En 1954, dans ses notes de mise en scène sur la troisième édition de l'Arlequin, Giorgio Strehler revient sur cette reprise d'une forme théâtrale évanouie pour en préciser le sens et le but et la détacher d'une simple reconstitution historique :

Il y a sept ans, notre Arlequin indiquait en Europe, à la fin d'une guerre sanglante qui avait généré pour tous son inévitable lot de souffrance et de désespoir, les retrouvailles avec quelques-unes des valeurs éternelles de la poésie et en même temps un message de confiance pour les hommes, à travers la libération du rire le plus franc, du jeu le plus pur. C'était le théâtre qui, avec ses acteurs, revenait (ou tentait de revenir) aux sources primitives d'un évènement scénique oublié au cours des vicissitudes de l'histoire et indiquait un chemin de simplicité, d'amour et de solidarité aux publics contemporains. C'était le théâtre qui redécouvrait (si l'on peut dire) une de ses époques glorieuses : la Commedia dell'arte, non plus comme un fait intellectuel mais comme un exercice de la vie contemporaine, en action. C'est ce qui fut peut-être le point qui distingua notre effort de celui de tant d'autres interprètes qui nous avaient précédés sur la même voie ${ }^{6}$.

La reprise de la Commedia dell'arte n'est pas pensée dans une optique de reconstitution historique, mais s'oriente vers la prise en compte d'un héritage et d'une mémoire destinés à s'incarner au présent et à échapper à la muséification. Elle s'inscrit dans une recherche théâtrale qui articule contexte politique d'élaboration d'une nouvelle société et 
volonté esthétique de poursuivre l'essence de la théâtralité en remettant au centre le jeu de l'acteur et le rire du public. Elle se propose ainsi de redonner espoir en l'avenir par le rire et par la monstration d'une collectivité solidaire, mettant en œuvre un travail théâtral quotidien.

\section{La reprise comme manifestation d'une recherche théâtrale au-delà de la reprogrammation}

7 L'Arlequin, dès la saison 1947-1948, fait l'objet d'une reprogrammation, liée à son succès et fait partie des spectacles envoyés en tournée afin de faire connaître ce tout nouveau théâtre qu'est le Piccolo Teatro et de lui donner une assise à la fois à l'intérieur de la péninsule et au niveau international. La reprise en tant que reprogrammation, en tournée ou au siège, fait partie du "prosélytisme culturel $»^{7}$ mis en place par Paolo Grassi, codirecteur plus particulièrement chargé de l'administration du théâtre. Elle contribue à augmenter le cercle d'initiés constitué par les premiers spectateurs du Piccolo Teatro tout en créant un lien, par le répertoire, entre les spectateurs. Mais le geste de reprise de l' Arlequin ne se limite pas à une reprogrammation destinée à conquérir un nouveau public.

8 Reprendre l'Arlequin pour Strehler, c'est aussi avoir l'occasion de proposer, de temps à autre, une nouvelle version de sa mise en scène, qui témoigne de sa propre évolution artistique. Cette recherche menée à l'intérieur d'une même œuvre transcrit un jeu entre le théâtre et le monde, qui entend réinventer la forme scénique en la confrontant au miroir de la société, dans un jeu de double qui répercute la structure profonde de la pièce. Au sein des sept versions ${ }^{8}$ signées par Strehler, il est possible de distinguer des cycles de reprise, guidés par l'approfondissement d'un même questionnement scénique.

premier cycle de reprises irait de la seconde à la quatrième version, menant une exploration et un questionnement de l'esthétique réaliste qui caractérisent à ce momentlà le travail du metteur en scène. La reprise de l'Arlequin est à comprendre en dialogue avec les mises en scène réalisées par Strehler à la même époque. Ainsi, la seconde version, créée le 17 avril 1952, est précédée par la mise en scène du Corbeau de Carlo Gozzi et de trois pièces de Goldoni : Les Amoureux, La Jeune Fille honorable (1949) et L'Amant militaire. La reprise de l'Arlequin permet de continuer le travail autour du masque, auquel Gozzi, dramaturge ennemi de Goldoni, accorde également un rôle prépondérant mais dans un sens différent. En effet, Carlo Gozzi rejette les perspectives de la réforme goldonienne qui, en s'appuyant sur le théâtre écrit et non sur le canevas, entend mettre en perspective le réel et condamne l'aspect bouffon et fantastique qui prévaut chez l'auteur du Corbeau et du Roi Cerf. Mettre en scène à plusieurs reprises l'Arlequin, c'est alors interroger les possibilités de réforme de la comédie et du théâtre italiens tout en tentant de retrouver le jeu all'improvviso, fondé sur les qualités d'imagination et de mémoire du comédien.

Lors de la seconde édition de la mise en scène, les fruits de ce travail de recherche autour d'une forme historique de jeu sont sensibles. Les masques ont été considérablement améliorés au fil des expériences. Réalisés par Amleto Sartori, ils sont désormais en cuir et constituent un appui de jeu au lieu d'une contrainte. Mais l'évolution décisive dans le geste de reprise concerne le travail dramaturgique et scénique que demande la Commedia dell'arte. Cette dernière est à l'origine une dramaturgie mixte, qui repose sur un scénario où, à partir du sujet et de la trame définis, s'entrecroisent quelques scènes entièrement 
rédigées, le développement (avec chute) des tirades et des dialogues et des moments improvisés, faits de lazzi et de bons mots.

11 Goldoni, dans la seconde version de son Arlequin qui est celle choisie par Strehler, transforme ce scénario en un texte intégralement rédigé, où sont indiqués assez précisément les lazzi, qu'il faut néanmoins réinventer et développer scéniquement: Arlequin tente de recacheter une lettre ouverte avec du pain, il déchire la lettre de change qu'on lui a confiée car il a besoin d'expliquer à un valet la disposition du dîner, il sert ses deux maîtres en même temps, mélange leurs affaires... Giorgio Strehler et Marcello Moretti, le premier Arlequin, réimaginent ces moments de liaison burlesques à partir des indications de Goldoni et en travaillent précisément le déroulement, qu'ils s'appuient sur une improvisation linguistique ou gestuelle, afin d'explorer les techniques de jeu de la Commedia. L'enjeu de cette première reprise est de redéfinir une forme historique tout en trouvant une liberté et une précision de jeu dans les contraintes d'un art codé par les masques, les emplois ${ }^{9}$, l'improvisation à partir du scénario et la structure de la troupe. Cette aspiration à la liberté est sensible dans la fuite par la salle d'Arlequin et par le fait de privilégier la conclusion de la seconde version de la pièce, qui propose une sortie de la fiction grâce à cette adresse au public :

L'homme est né pour les grandes actions ;

Son génie pourtant doit disparaître

Sous les traits de l'enfant Cupidon

À son pouvoir, je vais me soumettre ;

Je ne veux plus servir deux patrons,

Cher public, tu seras mon seul maître ${ }^{10}$.

Arlequin, démasqué dans ses fourberies mais délivré de ses maîtres, rejoint le monde, esquisse d'une prise en compte du réel et d'une mise en abyme de la fable et du dispositif théâtral. 
Arlecchino servitore di due padroni, de Goldoni. Mise en scène Giorgio Strehler, 1951-52

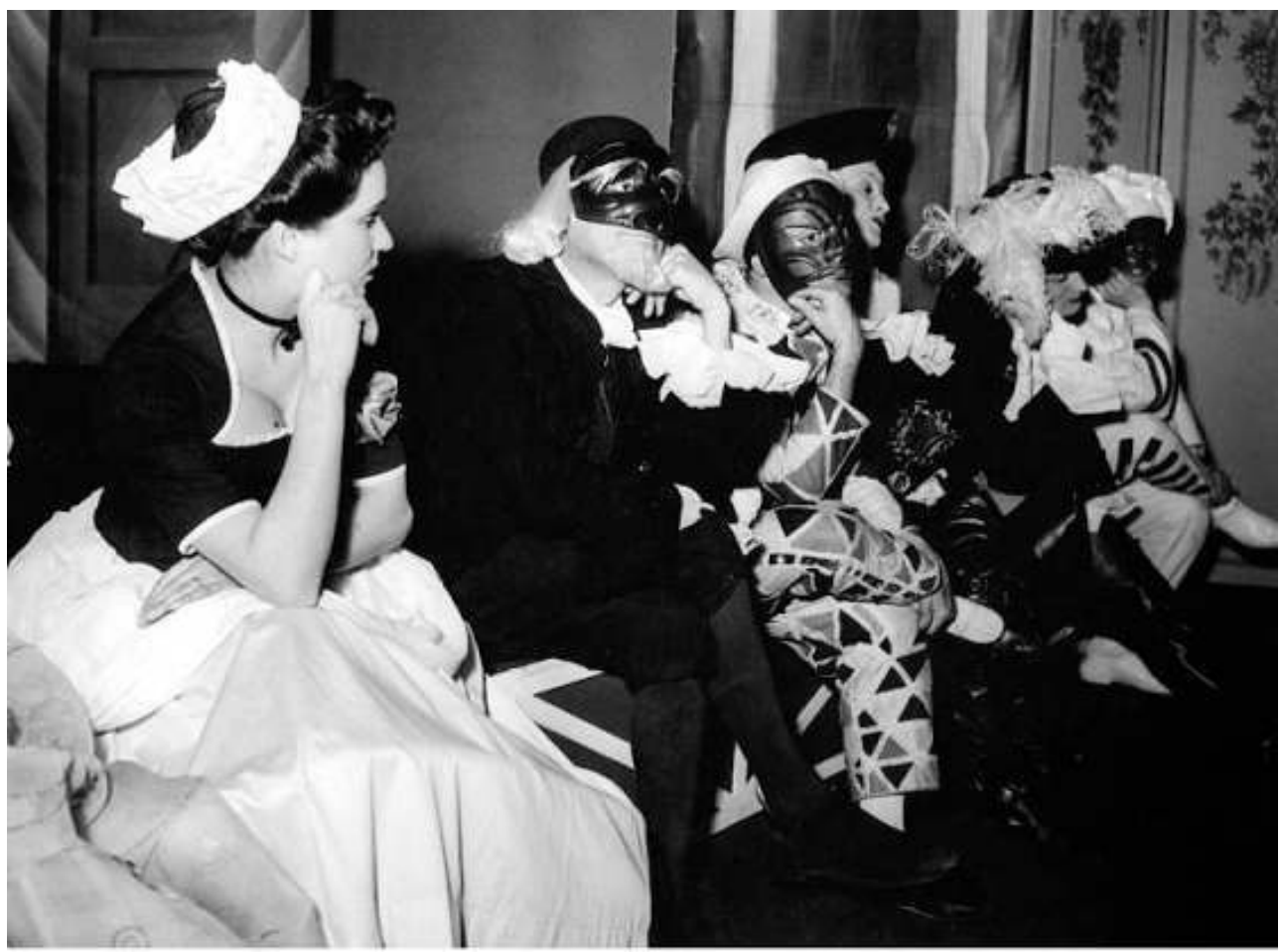

(C) Inconnu / Piccolo Teatro di Milano

La troisième version, celle du 27 août 1956, concrétise la conjonction entre la recherche $\mathrm{du}$ réalisme et les deux auteurs favoris du metteur en scène : Brecht et Goldoni. Elle correspond à un moment où Strehler souhaite éprouver les œuvres théoriques et dramaturgiques de Brecht et lier cette recherche à celle déjà entreprise sur Goldoni. Le metteur en scène, avant de rencontrer Brecht en 1955, au moment où il monte l'Opéra de Quat'sous, a lu toute son œuvre dramaturgique et théorique et voit en Brecht, comme en Goldoni, un réformateur observant une société en train de se transformer et saisissant la réalité de manière non mimétique. Il s'intéresse plus particulièrement au point de vue critique et à la possibilité effective de mettre en scène de manière épique. Dès lors, la mise en scène de l'Arlequin ne se concentre plus sur l'intrigue mais sur la représentation de la vie d'une troupe itinérante, afin de combiner le divertissement et l'interprétation critique de la Commedia, affirmant le regard brechtien et réaliste porté sur cette forme théâtrale. Dans une nouvelle scénographie signée Ezio Frigerio, l'estrade est remplacée par un tréteau positionné au centre du plateau, indice du théâtre dans le théâtre. Désormais, l'espace est double: il y a celui du tréteau, lieu du jeu et de la théâtralité propre à l'illusion, qui évoque la scénographie supposée de la Commedia ; et il y a celui, à vue, des coulisses et du reste du plateau, lieu du repos et de la préparation des comédiens mais aussi lieu des traces d'une histoire passée, dont il ne reste que des ruines. Ce second espace, constitué grâce à des pans de murs délabrés et aux chariots des comédiens, introduit une dimension historique qui fait ressentir le passage du temps, montrant une distance entre l'immédiateté du jeu et sa caractérisation, dans un second temps, comme les restes d'une tradition italienne qui tente de traverser les siècles. La perspective est à la fois réaliste et critique, à un moment où le néoréalisme cinématographique touche à sa fin. 
Arlecchino servitore di due padroni, de Goldoni. Mise en scène de Giorgio Strehler, 1959-60

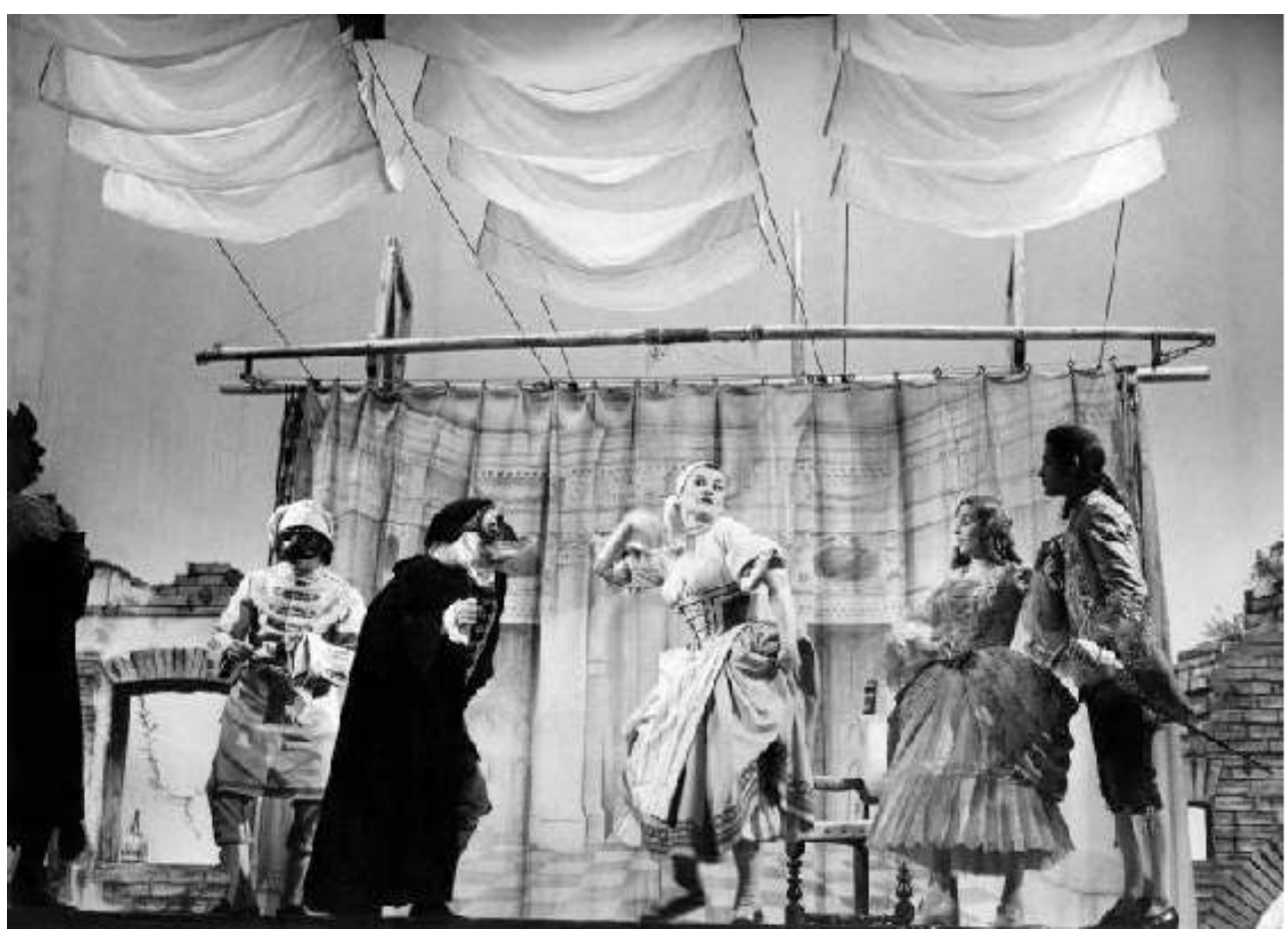

(C) Inconnu / Piccolo Teatro di Milano

Ce parti-pris est exploré avec des variations jusqu'en 1973, où la quatrième version offre un mouvement dialectique plus prononcé entre la théâtralité exacerbée du jeu sur l'estrade et le réalisme de la représentation de la vie quotidienne, fondée sur la présence des ruines et les échanges improvisés en dehors de l'estrade. Les variations, enrichies par l'expérience épique du metteur en scène, se nichent dans les détails et dans la contradiction entre des éléments jouant l'illusion (les détails soignés des toiles peintes, les meubles luxueux de la maison de Pantalon) et d'autres dénonçant l'artifice (le flottement de la toile, la cheminée peinte soulevée par Arlequin lors de son entrée). 
Arlecchino servitore di due padroni, de Goldoni. Mise en scène de Giorgio Strehler, 1972-73

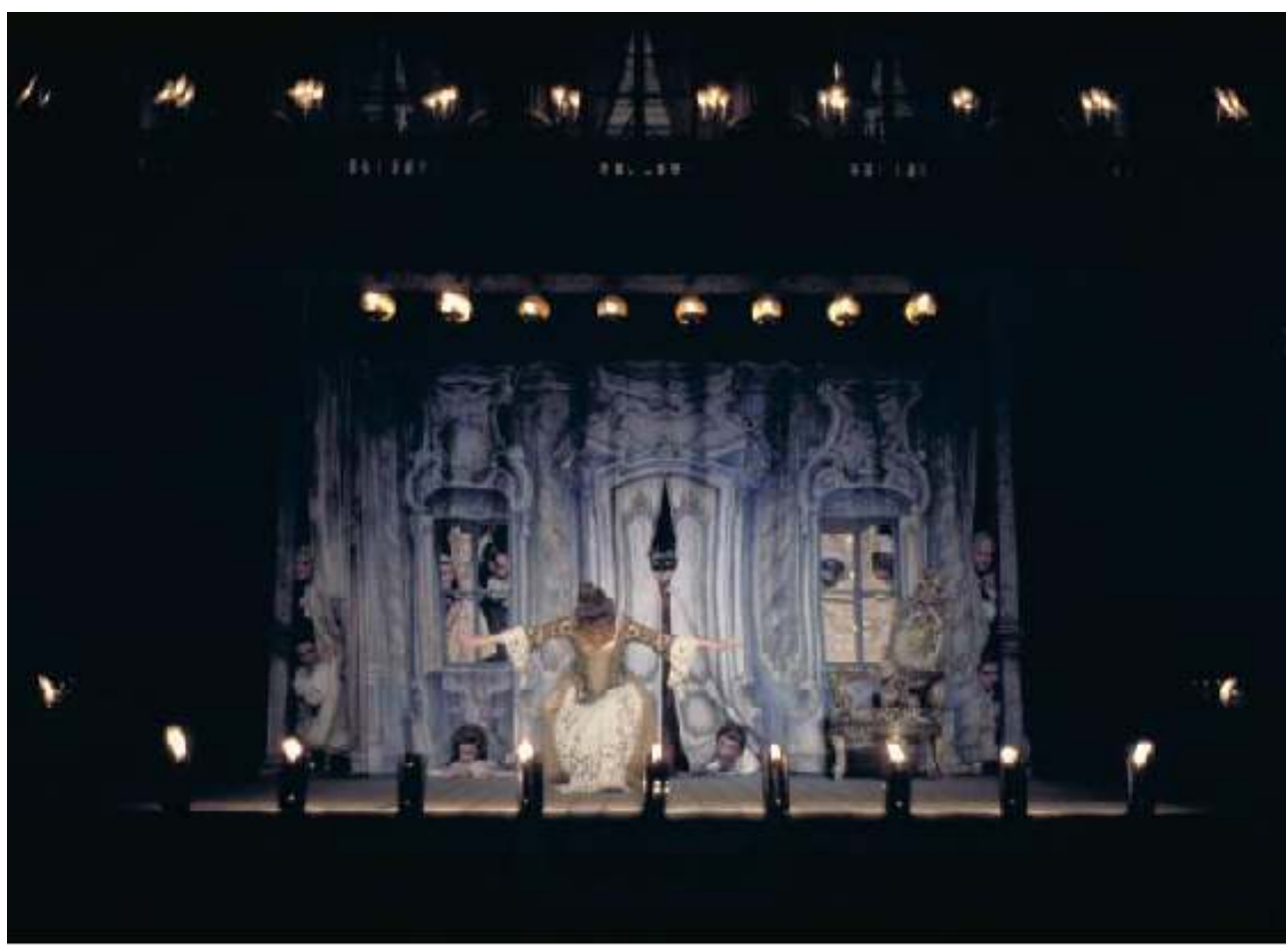

(C) LUIGI CIMINAgHI / PICCOLO TEATRO DI MILANO

Cette quatrième version, élaborée au moment du retour de Strehler à la tête du Piccolo Teatro, préfigure la seconde dynamique de mise en scène de la pièce goldonienne, fondée sur la conscience d'un effacement mélancolique du théâtre et de la peur de sa disparition, perceptible dans l'édition suivante, donnée en 1977. La cinquième édition, dite édition de l'Odéon car imaginée spécialement pour le théâtre parisien, constitue un déclic, qui trouve un écho dans le travail que Strehler mène autour de l'illusion à cette période. Cette dynamique crépusculaire trouve sa plus grande expression dans La Tempête, montée à la fin de la saison 1977-1978 et surtout en 1984 avec L'Illusion comique. Pour Strehler, cette réinvention mélancolique est déterminante, comme en témoigne la reprise du travail esquissé à l'odéon, une fois la compagnie rentrée en Italie :

C'est une édition que nous aimons particulièrement, parce qu'elle a été surtout l'occasion d'un approfondissement sur le versant du contrechant des comiques qui font et assistent au spectacle de l'Arlequin serviteur de deux maîtres. Le début de chaque acte, les finals et beaucoup d'idées, d'inventions, de suspensions mélancoliques, un certain jeu du théâtre dans le théâtre, un journal de la vie des artistes comiques dans un siècle merveilleux et lointain, appartiennent seulement à cette édition française. Comme si de l'avoir fait naître et vivre sur un plateau français, dans un théâtre tant aimé et si riche d'histoire, comme l'odéon, si plein de fantasmes théâtraux très anciens, avait en quelque sorte imposé à notre imagination la nécessité de renouer avec certaines de nos racines européennes, avec l'histoire des comédiens italiens au cœur du XVII ${ }^{e}$ et du XVIII ${ }^{\mathrm{e}}$ siècle à travers toute l'Europe pour y porter le théâtre, mais aussi pour lier les hommes entre eux, pour créer quelques histoires mystérieusement profondes, et qui ont perduré dans le temps, comme des graines d'humanité, de vitalité et d'imagination de la grande culture européenne ${ }^{11}$. 
L'approche de la pièce se fait ainsi plus mélancolique, soulignant une perte de confiance dans la capacité du théâtre à survivre. Elle introduit une distance critique en infléchissant la spontanéité et la vitalité du jeu vers une réflexion sur la transmission, la vie des comédiens et traite l'Arlequin comme une matière désormais sortie de sa période de gloire et trace d'un passé que notre époque a du mal à saisir et à réinventer.

Arlecchino servitore di due padroni, de Goldoni. Mise en scène de Giorgio Strehler, 1977-78

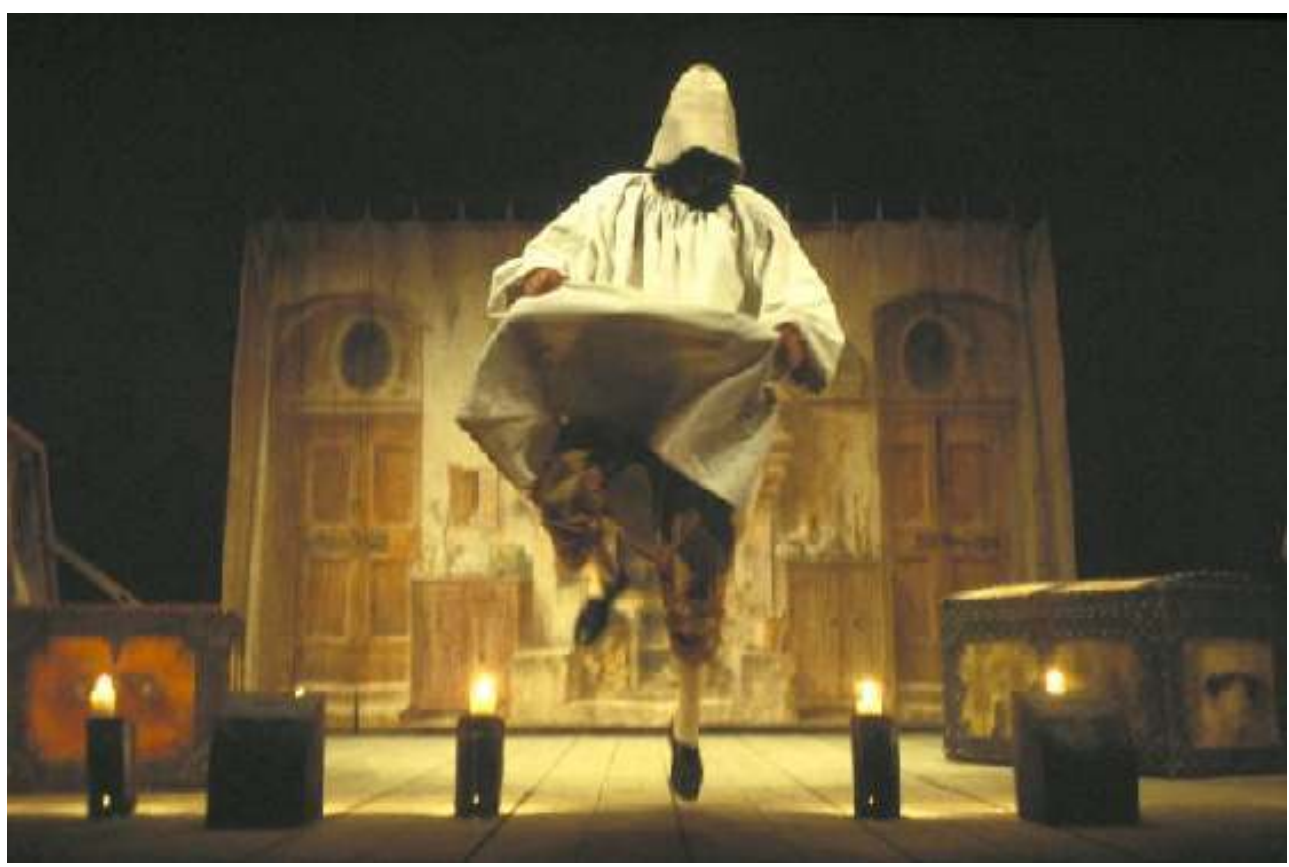

(C) LUIgI CIMINAgHI / PICCOLO TEATRO DI MILANO

Le tréteau, centre de la scénographie du précédent cycle, disparait pour laisser place à un plateau nu aux coulisses à vues, éclairé en apparence seulement par des quinquets en avant-scène. Des tentures représentent l'auberge et la maison de Pantalon mais laissent la plupart du temps place à un fond neutre, qui fait ressortir les jeux de scène poussés jusqu'au cabotinage, tout en conservant le jeu attaché à la représentation de la compagnie en train de donner un spectacle. Les acteurs, une fois leurs répliques dites, se tiennent en bord de scène et commentent, se remaquillent, écoutent la scène ou révisent à voix intelligible leurs répliques, détournant le spectateur de l'action principale, désignée comme un prétexte. Ils dévoilent au spectateur les artifices du théâtre, comme celui consistant à recréer un canal vénitien grâce à une chandelle et une bassine et établissent une forme de continuité, une zone floue entre la vie et la convention qui dénonce l'illusion. Le théâtre se trouve à la fois exalté et mis en doute, montré dans la fragilité de son artisanat, dans un écho aux inquiétudes de Strehler, présentes depuis son retour, seul, à la direction du Piccolo Teatro, en 1972. L'heure ne peut plus seulement être à la fête et la fuite impossible d'Arlequin, par la salle comme par la scène, le rideau refusant de se relever, n'est résolue in extremis que par une envolée dans les cintres sur un nuage héritier du théâtre à machines, dans une déification ironique et une allusion à l'héritage technique de Nicolà Sabbattini et Giacomo Torelli. L'apothéose d'Arlequin se mêle à sa relégation dans l'histoire théâtrale et signe la difficulté pour le théâtre de continuer à exister en tant qu'artisanat et en tant qu'héritier d'une tradition théâtrale qui semble désormais incongrue, jusque dans ses artifices. 


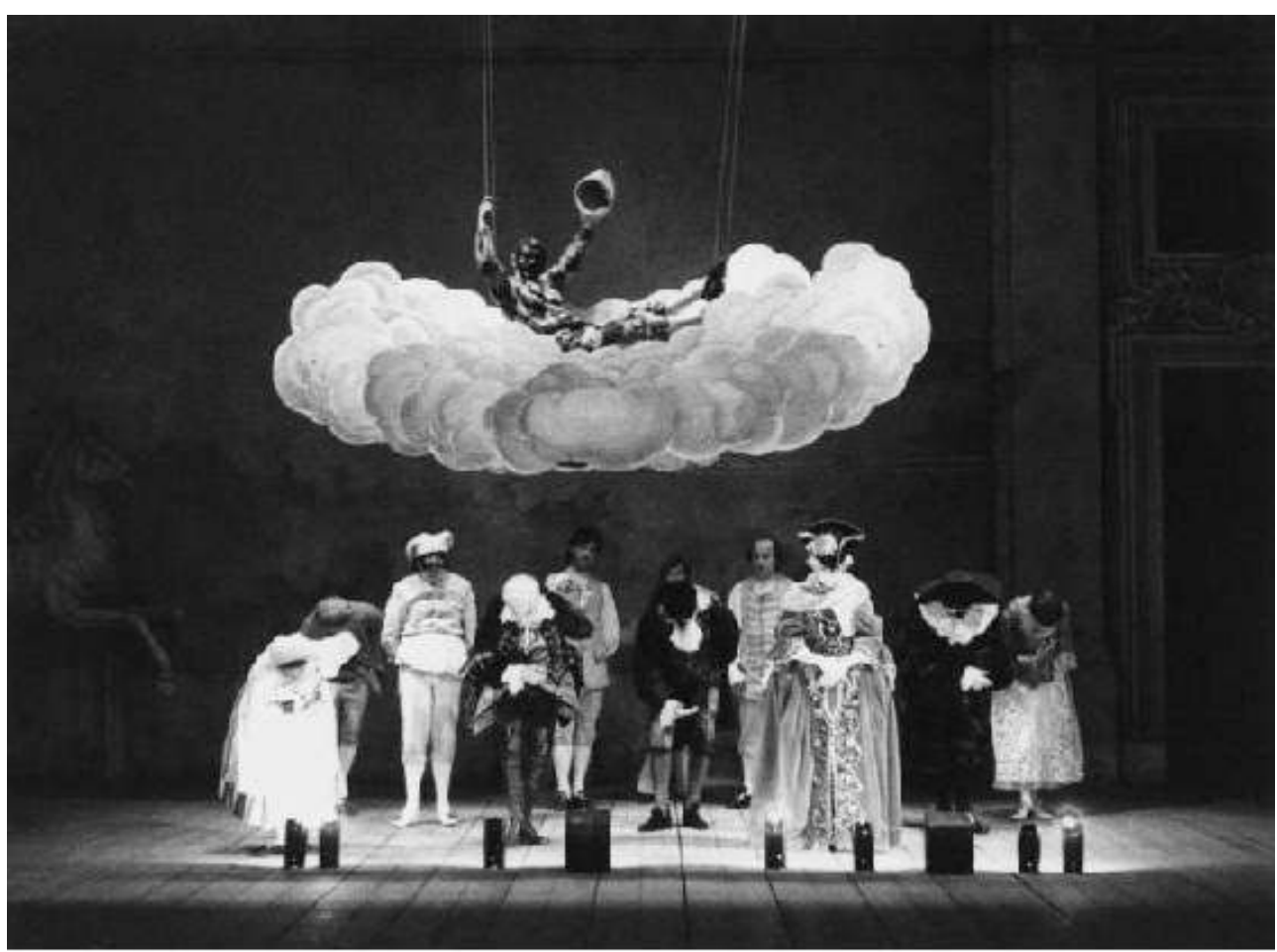

@ LUIGI CIMINAgHI / PICCOLO TEATRO DI MILANO

Deuxième variation autour de cette perspective mélancolique, l'édition de 1987 dite de l' Adieu, accentue la lassitude qui envahit le plateau en voilant le plaisir et l'énergie du jeu, et fait transparaître les conflits et les difficultés que le metteur en scène rencontre avec les travaux du théâtre et la municipalité de Milan. L'impulsion de la recherche et de la réinvention artistique s'est usée. La reprise relève de l'obligation, celle de l'anniversaire des quarante ans du Piccolo Teatro mais cherche son sens et ne se justifie plus que comme un dernier salut à une expérience fondatrice. Strehler, dans sa note d'intention, parle de " témoignage » et de «fidélité à ce qui a accompagné durant quatre décennies leur vie de gens de théâtre ${ }^{12}$. C'est un geste d'adieu, qui vient clore une aventure théâtrale et qui précède la démission de Strehler. Le parti-pris de cet Arlequin devait être la réunion, grâce à un système d'alternance, de tous les comédiens qui ont participé aux différentes mises en scène. Il n'est réalisé qu'en partie, à cause des indisponibilités ou de la disparition de certains comédiens mais l'intention est présente grâce à une distribution différente chaque soir, durant tout le temps des représentations. Mais malgré cette présence du collectif et de la pérennité de l'œuvre d'art,

Les acteurs de l'Arlequin 1987 sont fatigués. Ils ont le droit et le destin d'être fatigués. Certains d'entre eux portent ce spectacle depuis des décennies, d'autres l'ont laissé pour ensuite le reprendre successivement. Cependant ils ne montreront pas leur fatigue [...]. Parce que c'est précisément là que se trouve notre extrême dignité de serviteur du théâtre et le signe de notre humilité, portée comme un signe d'honneur. Cette soirée, ces soirées auront donc certainement leur poids de tristesse et de consentement à la vie qui passe. Et dans le jeu hilarant de l'Arlequin serviteur de deux maitres, il ne peut manquer d'y avoir le frisson et l'ombre du temps ${ }^{13}$. 


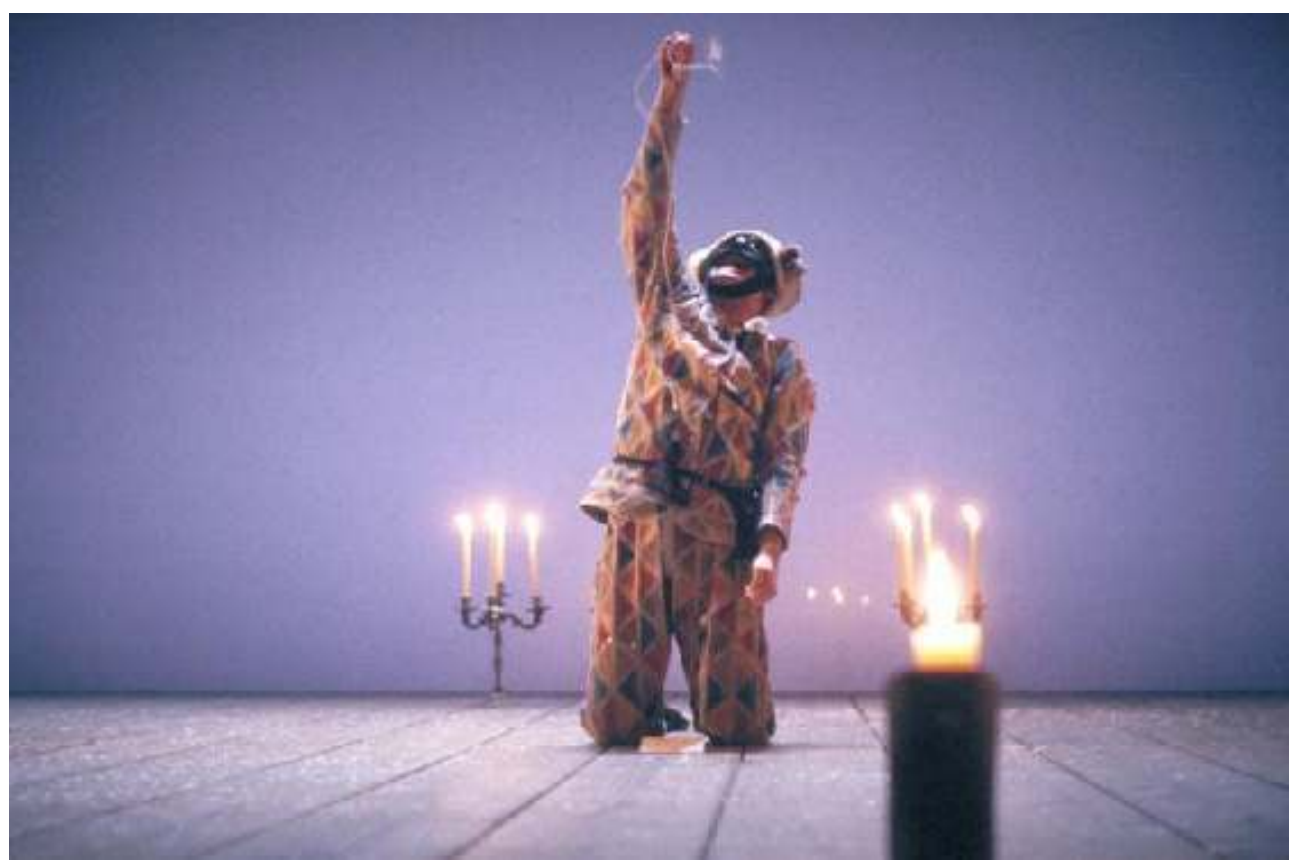

Arlequin (Ferrucio Soleri) dans le Lazzo de l'enveloppe et du pain, 1987

EDITION DE L'ADIEU ( $\subset$ LUIgI CIMINAgHI / PICCOLO TEATRO DI MILANO

La reprise devient le signe de la fin d'un parcours au sein du théâtre et de la fin d'un monde, soulignant l'érosion irrépressible du temps, que le jeu ne peut plus divertir et alléger. Cette menace de disparition se traduit par un dépouillement extrême de la scénographie : une rampe de quinquets, quelques candélabres au sol qui aident parfois à figurer les portes, un fil tendu à mi-hauteur sans qu'un rideau (brechtien) vienne s'y glisser et où pend un petit ruban comme oublié, une toile de fond transparente propice aux ombres et quelques accessoires indispensables (les malles, les petites tables, les paravents) viennent situer l'intrigue et servent de point d'appui au jeu. L'édition de l' Adieu se révèle propice à l'autocitation, qu'elle se manifeste dans la note d'intention avec une conclusion évoquant l'adieu de Lioubov dans La Cerisaie ou qu'elle intervienne dans le cadre d'un théâtre dans le théâtre, qui voit les acteurs investir l'avant-scène et les premiers rangs pour observer leurs camarades et commenter les accidents. Ainsi, un grand coup de vent et de pluie traverse le théâtre et les acteurs murmurent : « la tempête, l'orage... ", citation inattendue des derniers succès du metteur en scène ${ }^{14}$.

Laissant à la marge l'intrigue d'Arlequin, Strehler construit par petites touches une réflexion acerbe sur le temps qui passe et l'approche de la mort, superposant les strates, jouant des oublis d'accessoires et de l'irruption des souvenirs: Pantalon s'interrompt soudain et se rappelle du bon vieux temps et des soirées passées en bonne compagnie. Prisonniers du temps qui exerce son usure, les acteurs feignent de bafouiller, le costume d'Arlequin a perdu de son éclat, la figure du souffleur tourne à celle du vieux grincheux qui dort et marmonne et au salut, dans un dernier memento mori adressé au public, les acteurs les plus âgés ôtent leurs masques et leurs perruques, dévoilant le passage du temps et la réalité de la compagnie. Cette édition de l'Adieu est celle d'une intrigue parasitée par les traces, qui freinent l'énergie des stratagèmes et transforment par 
moment les acteurs en fantoches pris dans la répétition de leur rôle, perdus sur un plateau qui désespère de retrouver l'enfance de l'art.

Arlecchino servitore di due padroni, de Goldoni. Mise en scène de Giorgio Strehler, 1986-87

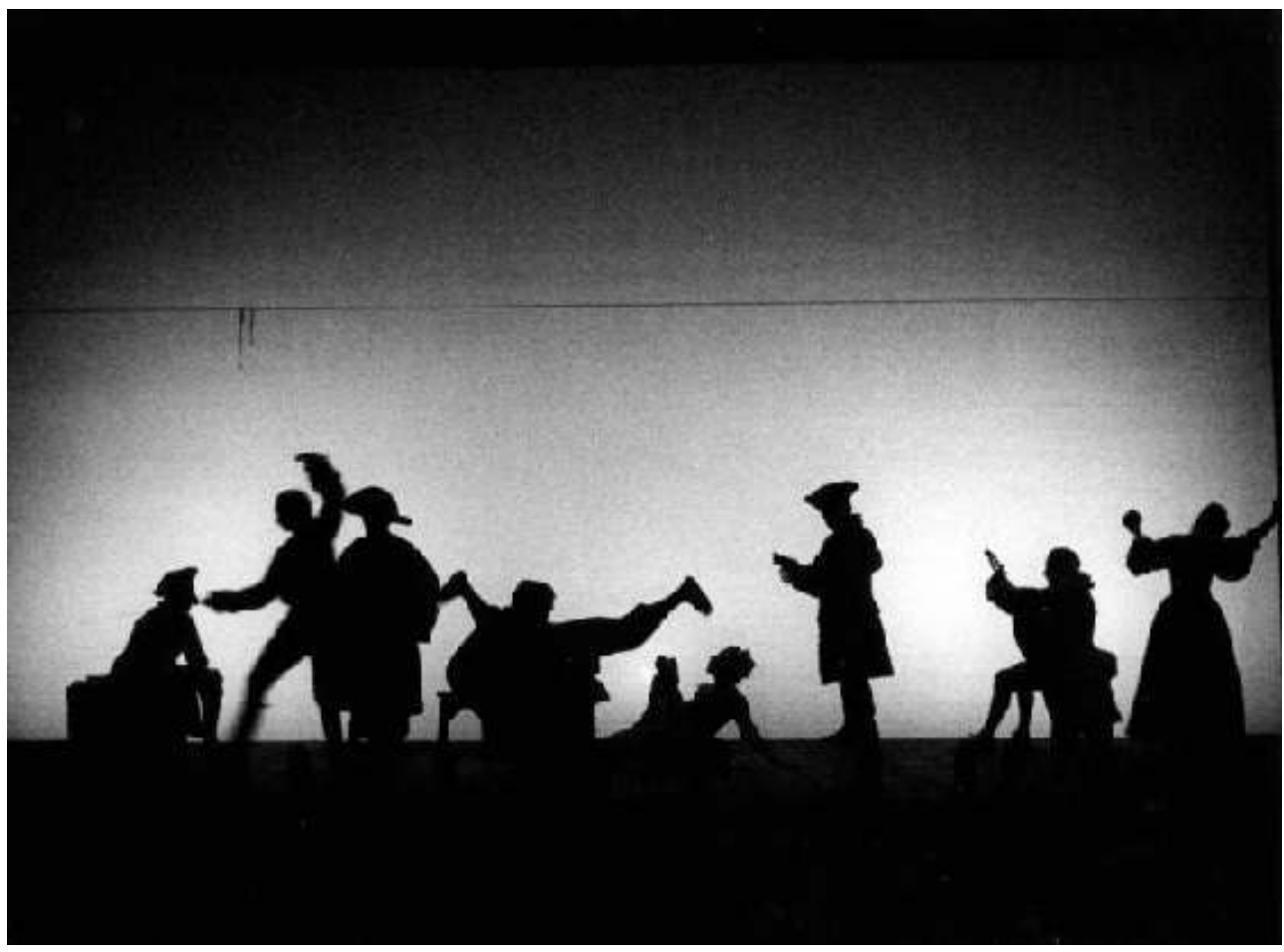

EDITION DE L'ADIEU

(C) LUIGI CIMINAgHI / PICCOLO TEATRO DI MILANO

21 Lors de ces deux cycles de reprises, l'un d'approfondissement du réalisme, l'autre de questionnement de l'illusion et de la fonction du théâtre, l'esthétique scénique a été sans cesse soumise à variations, sous la double contrainte de l'évolution artistique du metteur en scène et du changement de la place du théâtre au sein de la société. Mais en arrièreplan, s'est jouée une autre fonction de la reprise, liée à une tradition scénique qui tisse un pont entre la Commedia dell'arte et le théâtre oriental. Non plus celle de la mise à l'épreuve de l'inventivité et d'un regard critique, mais celle de la transmission d'une tradition scénique et culturelle, qui s'effectue à l'échelle du rôle-titre comme à celle de la troupe.

\section{La reprise à l'épreuve du corps et du jeu de la transmission}

Si cette transmission est une conséquence de la reprise régulière du spectacle et de l'indisponibilité de certains acteurs, elle prend forme dans l'édition dite du «Buongiorno » de 1990 où Ferruccio Soleri, le second titulaire du rôle-titre, partage la scène et son expérience avec les élèves du cours Jacques Copeau de l'École du Piccolo Teatro, dont cette version constitue le spectacle de sortie. Lien physique avec l'histoire théatrale italienne, la reprise est un outil de formation, qui inscrit les jeunes acteurs dans une histoire du jeu italien et dans l'histoire du Piccolo Teatro en tant qu'institution fondatrice du théâtre public au sein de la péninsule. Comme le souligne Ferruccio Soleri, il s'agit de transmettre 
une technique de jeu, dotée d'une grammaire scénique spécifique, qui s'appuie sur un travail de recherche historique, dont les éléments sont ensuite expérimentés au plateau :

Tout est le fruit d'une technique bien précise. Arlequin est un personnage stylisé, un masque qui a ses rythmes de mouvements et de jeu, des rythmes ternaires [...]. Il y a des positions fixes, désormais " catéchisées ", soit par moi, soit par Strehler puis recréées, réinventées. [...] La tradition de la Commedia dell'arte s'est interrompue au $\mathrm{XIX}^{\mathrm{e}}$ siècle. C'est pourquoi nous avons dû la reconstruire, en partie grâce aux iconographies, en partie grâce aux chroniques de l'époque. Nous avons pensé que certains mouvements et pas étaient caractéristiques de l'Arlequin de l'époque et nous les avons conservés, fixés ${ }^{15}$.

Une fois la partition établie et de la même manière qu'a eu lieu la transmission entre Marcello Moretti et Ferruccio Soleri, d'abord sur le mode de la copie puis de l'appropriation, les jeunes acteurs commencent par répéter les lazzi et les situations de la comédie sous le regard des anciens. Puis, ils se les approprient progressivement, introduisant leur propre variation à l'intérieur de la tradition et livrant, sous le contrôle du metteur en scène, leur interprétation personnelle de leur rôle et d'une forme théâtrale historique. Car au-delà de la transmission d'une technique et d'une tradition codifiées, l'enjeu, pour Strehler, est désormais d'apprendre à ces jeunes acteurs une manière de concevoir leur métier et d'être au monde: "Nous voudrions que cet apprentissage du théâtre serve aussi pour la vie. Que celui qui ne fera pas du théâtre son métier n'ait pas perdu en vain ces années ${ }^{16}$. " L'essentiel est qu'ils gardent trace et défendent un état d'esprit, une discipline intérieure envisagée comme un rempart à la société de consommation, dans un mouvement de réaction dont la dynamique remonte à Jacques Copeau, maître revendiqué de Strehler. Il s'agit alors de transmettre un héritage théorique et esthétique grâce au procédé de la reprise et de renverser la force négative de "l'horrible machine à monter des spectacles ${ }^{17}$ ", qui pourrait se nicher dans les tournées et la reprogrammation de la mise en scène jusqu'à l'épuisement pointé par Strehler en 1987.

Dans la version de 1990 comme dans celles de 1993 et de 1997 qui relèvent à nouveau de l'hommage, il y a la volonté de multiplier les compagnies de l'Arlequin, afin de les mélanger, de jouer l'alternance et de privilégier la différence entre les représentations, y compris à l'intérieur d'un même spectacle. Cette prolifération de la reprise veut mettre en valeur l'acte collectif de la mise en scène et recherche une fusion des différentes distributions, où le spectateur ne pourrait plus distinguer qui joue à tel ou tel moment du spectacle. La répétition du geste de reprise fait du spectacle une matière mise en commun, qui sert à révéler le collectif et l'unité profonde qui se jouent à l'intérieur de la mise en scène. L'idéal que Strehler cherche à atteindre est un impératif esthétique et moral : «Se fonder sur la théâtralité, - acteurs et public - pour devenir une entité collective qui parle d'une seule voix ${ }^{18}$.»

C'est pour parvenir à cette dimension collective qu'il faut sans cesse reprendre le spectacle, en le réinventant et en en conservant l'esprit et la leçon, celle d'une tradition codifiée, mise au miroir de l'époque contemporaine et de l'évolution théâtrale. Éloignée d'un geste de recyclage ou de la réédition pour usage commercial d'un produit épuisé, la reprise-variation de l'Arlequin est pensée comme un exercice de communauté et de mise en commun, qui s'oppose idéologiquement à une société de consommation privilégiant l'individu et la duplication industrielle. L'Arlequin présente ainsi la marque d'une réflexion plus générale de Strehler sur la nécessité de mettre en valeur le répertoire, seule trace certaine de son œuvre et moyen de dialoguer avec le public. La reprise, dans 
sa fonction de répétition et de variation, concrétise ce dialogue en explicitant les questionnements de Strehler sur ce qui survit de l'art théatral quand ce dernier se trouve menacé : les pouvoirs du jeu sont-ils toujours intacts? Peut-on réinventer à l'infini une forme sans l'épuiser? La reprise de l'Arlequin témoigne de cette volonté d'éprouver le théâtre, dans son essence comme dans son évolution.

Arlecchino servitore di due padroni, de Goldoni. Mise en scène de Giorgio Strehler, 1990-91

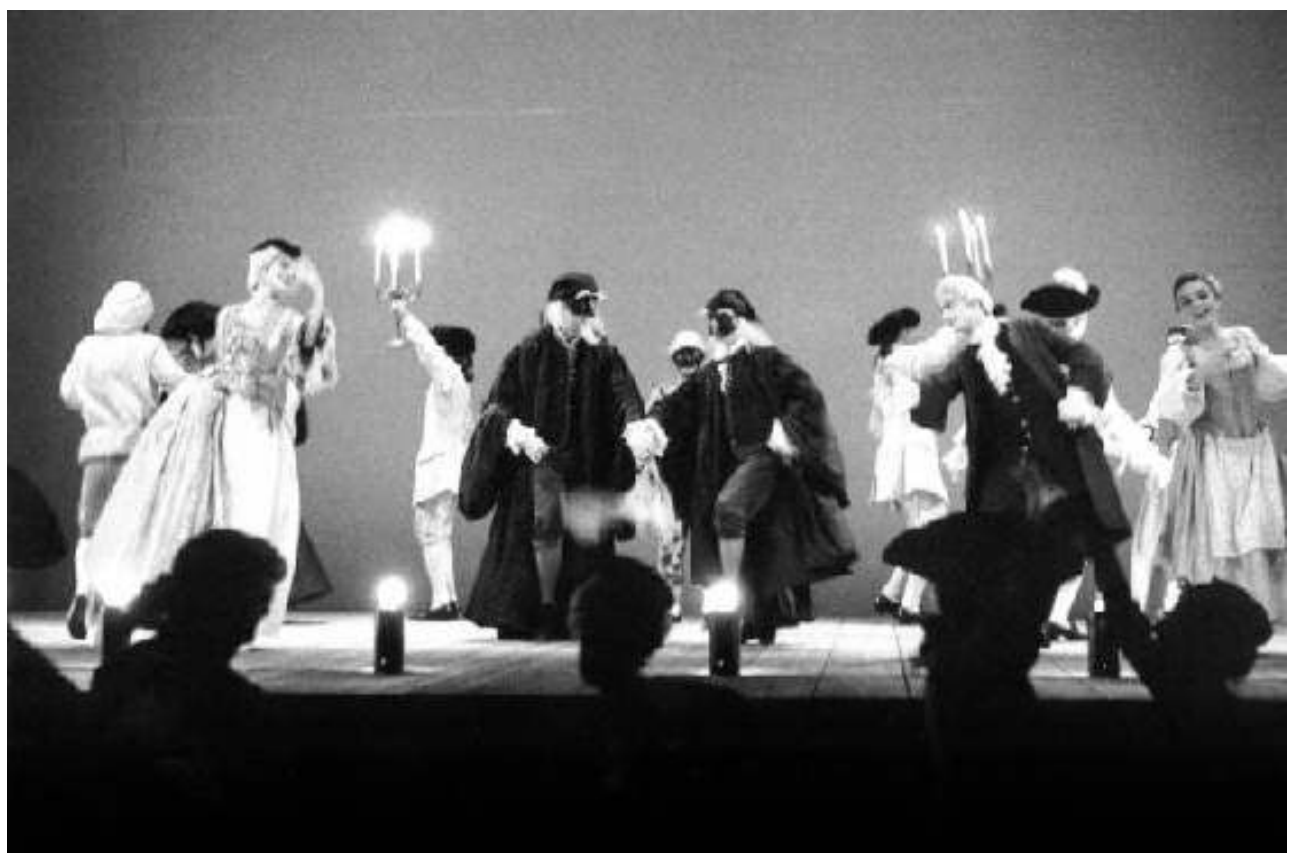

(C) LUIgI CIMINAgHI / PICCOLO TEATRO DI MILANO

\section{De la mémoire vivante à la dimension patrimoniale}

Partie prenante d'une réflexion sur la survie du théâtre, la reprise entend lutter contre l'éphémère en constituant une mémoire et une pratique vivantes, inédites dans les habitudes de création contemporaine où les acteurs passent de plus en plus d'une production à l'autre et n'ont pas l'occasion d'explorer les possibilités d'un rôle sur plusieurs années. Pour Strehler, la pérennité de cette mise en scène réalise ce vers quoi devraient tendre les œuvres d'art :

Mais est-ce exact, de définir comme extraordinaire la traversée des années et même des siècles pour une œuvre théâtrale, pour n'importe quel geste poétique des hommes? N'est-ce pas ou ne devrait-ce pas être au contraire la caractéristique de l'œuvre d'art, en tant qu'œuvre d'art? La véritable œuvre d'art parle avec des accents différents, avec des perspectives différentes mais toujours contemporaines au public auquel elle s'adresse ${ }^{19}$.

Elle est le signe d'une recherche artistique en mouvement mais déploie également une dimension métaphysique, la reprise devenant le lieu et l'occasion d'une méditation sur la vie, quotidienne et théâtrale, resserrant les liens entre les deux mondes dans un mouvement dialectique qui fédère la pensée théâtrale de Strehler.

Ainsi, cet Arlequin éternel est le signe de la vie qui passe et se renouvelle. C'est le sang qui bat et court dans les veines d'un théâtre réel et imaginaire, comme dans un corps humain. Du reste, le spectacle du Piccolo Teatro, de l'Arlequin serviteur de deux 
maîtres, sous ma direction, ma mise en scène, a connu des éditions différentes, parfois semblables, parfois totalement différentes, non dans l'esprit mais dans la forme, de façon que le public, au moins tous les cinq ou six ans, voit un autre spectacle, non la copie du précédent, non son contraire, mais sa continuité dialectique, identique et différente dans le même temps. Comme la vie ${ }^{20}$.

Dépassant la réflexion spirituelle, la reprise du spectacle est liée au statut d'emblème qu'a très vite acquis la mise en scène, devenant la carte d'identité du théâtre lors des tournées. Elle fait connaître le théâtre à l'échelle internationale et affirme son identité en associant étroitement, sous le signe de "l'italianité ", une histoire culturelle et une histoire scénique jusque-là peu connues dans son actualité. Elle maintient vivant l'héritage théâtral d'une forme artistique et offre aux spectateurs du monde entier une image concrète de ce qu'est la Commedia dell'arte lorsqu'elle rencontre un auteur majeur du répertoire italien. La reprise ne fait pas alors œuvre d'invention scénique mais donne vie à une mémoire, en en respectant les caractéristiques et les contraintes. Son sens change selon l'échelle et le contexte qui viennent l'accueillir. À l'échelle de Milan ou des théâtres stables italiens qui programment régulièrement le spectacle dans ses différentes versions, elle est une manière de lutter contre l'éphémère du théâtre et de constituer une mémoire commune aux spectateurs, qui fait dialoguer les générations et tisse un fil directeur à l'intérieur des créations de Strehler. Elle appartient au répertoire que Strehler a choisi d'approfondir, celui qui, structuré essentiellement autour de Brecht et de Goldoni ${ }^{21}$, questionne un geste réformateur du théâtre, que le metteur en scène souhaite à son tour accomplir :

[Pour Brecht comme pour Goldoni], le cadre de leur travail est une société saisie dans un moment de difficulté : pour Goldoni, celui du passage à un ordre et des habitudes bourgeoises; pour Brecht, le moment où le libéralisme apparu au XIX ${ }^{\mathrm{e}}$ siècle affronte, dans notre siècle, sa première crise violente ${ }^{22}$.

Pour Strehler, son geste de réforme se heurte à la tendance à la festivalisation du théâtre et à l'individualisation de la société, geste qui ne correspond plus au projet de société homogène de l'après-guerre, désormais inatteignable. Mais il souffre aussi de l'échec du rêve d'un théâtre continuellement in progress, révolutionnant la vie et l'art théâtral. Le rêve de communauté unie, mis en doute puis abandonné au cours des années 1960, Strehler avait envisagé cette transformation constante du théâtre à travers la mise en œuvre des idées brechtiennes. Elles lui permettaient d'envisager un art qui, certes, divise le public mais parvient à s'inscrire dans une recherche permanente, confirmant la nécessité de la reprise comme variation. Mais cette dynamique s'épuise à son tour à partir de 1972. Strehler abandonne peu à peu la confrontation entre le théâtre et le monde pour se concentrer sur la variation des possibilités scéniques et de l'illusion théâtrale, qui accueille désormais le monde et y questionne sa place. La reprise de la pièce de Goldoni propose désormais, à partir de 1987, une variation en mode mineur, moins directement en prise avec l'époque.

Dans le même temps, à l'échelle internationale, l'Arlequin devient la reprise d'un succès théâtral divertissant, représentatif de l'esthétique du Piccolo Teatro et de ce qui est attendu du théâtre italien par les programmateurs étrangers, même si la tournée peut être l'occasion d'une recréation, comme lors de la version de l'Odéon. La réflexion esthétique laisse place à la volonté d'élargir l'audience du théâtre et entraîne le spectacle sur la voie de l'histoire, du patrimoine et même du record, de par la longévité d'exploitation du spectacle qui transforme la reprise en performance statistique. 
31 Après la mort de Strehler, le spectacle s'inscrit dans la ligne de programmation historique et patrimoniale du Piccolo Teatro désormais dirigé par Sergio Escobar et Luca Ronconi. L' Arlequin entre dans le cadre de la reprise de certaines mises en scène de Strehler afin de donner à voir le patrimoine du théâtre et de conserver le lien avec l'esprit humaniste et civique qui a présidé à la fondation du théâtre. Le principe qui conditionne la reprise est simple et identique pour chaque mise en scène qui en fait l'objet : il faut qu'au moins un interprète ait participé à la mise en scène initiale. Dans le cas de l'Arlequin, la continuité est assurée par Ferruccio Soleri, qui joue Arlequin depuis 1963 et depuis 2002, alterne la tenue du rôle avec son apprenti Enrico Bonavera, également interprète du rôle de Brighella. Mais, malgré cette continuité, l'absence de Strehler ainsi que la programmation du spectacle, à partir de 2010, à l'approche de la fin d'année et des fêtes de Noël signent l'entrée de la mise en scène dans une dimension qui conjugue célébration festive et patrimoine. La dynamique de recherche théâtrale a disparu et le spectacle est désormais la trace d'une tradition singulière du Piccolo Teatro, celle de reprendre certaines mises en scène après la mort de leur auteur. L'Arlequin serviteur de deux maîtres devient un lieu de mémoire, au sens d'hommage et de lien entre le passé, le présent et l'avenir de l'institution. Cette reprise est un hommage qui maintient artistiquement vivante la présence de l'ancien directeur du Piccolo Teatro au sein du théâtre. Elle est un espace d'expression qui témoigne d'un geste artistique qui a sans cesse réinventé et mis en perspective, comme un emblème, une des sources du théâtre italien. La représentation de l'Arlequin devant plusieurs générations de spectateurs, crée entre eux, selon le fantasme de Strehler, un lien virtuel qui combat le caractère éphémère de la scène et lui offre une permanence à l'abri de la pétrification. L'importance de ce lieu de mémoire, perçu sur le mode de la nostalgie, de la remémoration ou de la découverte par les spectateurs, est fortifiée par la part économique. Le spectacle, grâce à son aura, affiche souvent complet. Réclamé par les spectateurs et garant de l'histoire et de l'évolution du Piccolo qu'il accompagne tel un point de repère, il constitue un succès de programmation assuré pour le théâtre, ce qui, dans une période de réduction drastique des subventions initiée en 2006 et amplifiée depuis 2010, aide le Piccolo Teatro à rester autonome et à consacrer plus des deux tiers de son budget à la production artistique.

Reprendre l'Arlequin serviteur de deux maîtres était pour Strehler une manière d'ériger la variation en méthode de travail et d'élaborer un répertoire étroitement lié à son projet de réforme théâtrale et à sa manière d'articuler le théâtre et le monde, qui tente de retravailler le passé théâtral tout en imaginant l'avenir, dans la forme et dans la transmission. À ces dimensions de mémoire vivante et d'invention scénique, vient s'ajouter la rançon, parfois bienvenue, parfois pesante, de la reprise : celle du succès et de la muséification, qui réduisent la recherche artistique à sa commercialisation ou à sa conservation, la rendant étrangère à l'évolution du monde et des formes théâtrales. 
Arlecchino servitore di due padroni, de Goldoni. Régie de Giorgio Strehler et mise en scène de Ferruccio Soleri, 2003-2004

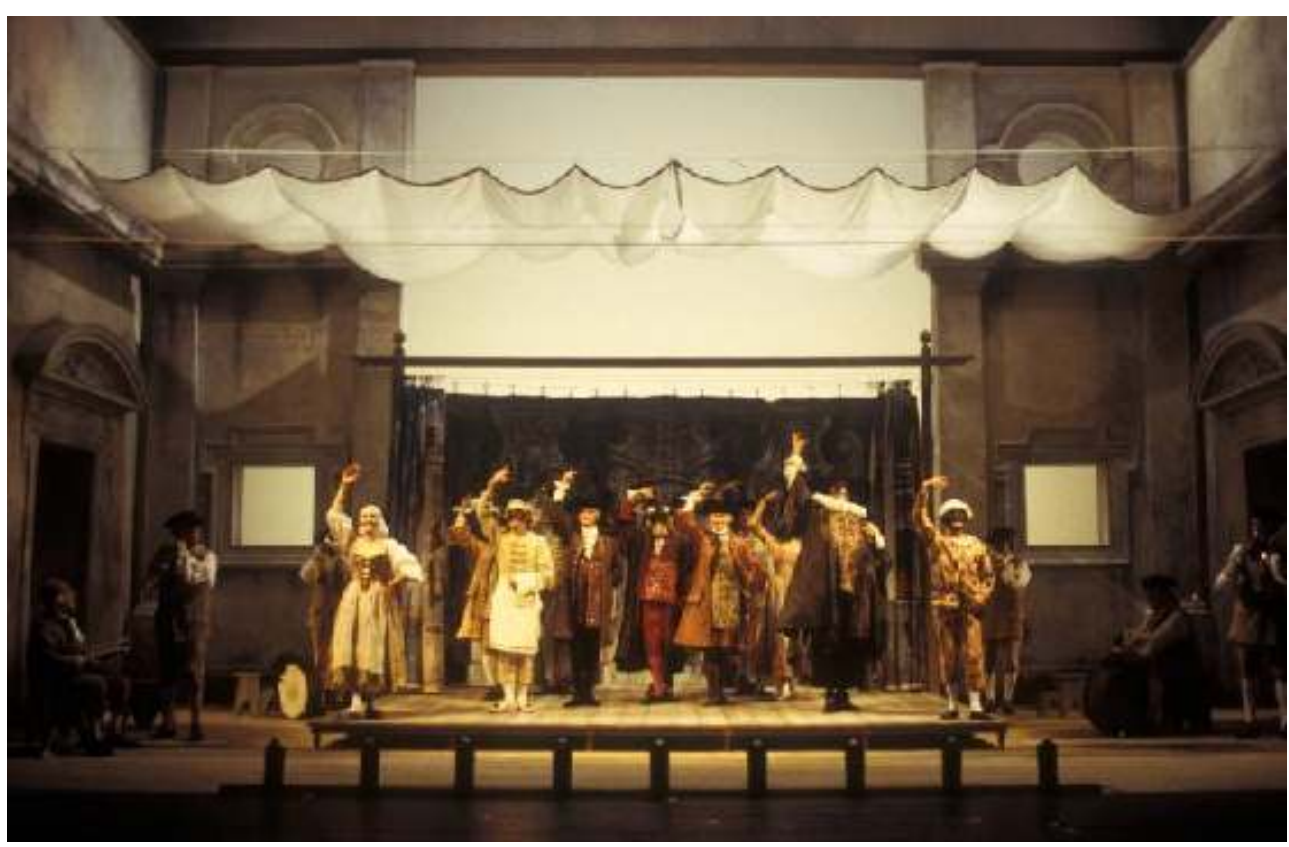

Le salut de la compagnie.

(c) Diego et Luigi Ciminaghi

\section{NOTES}

1. Le Piccolo Teatro de Milan, créé le 14 mai 1947 à l'initiative de Giorgio Strehler et Paolo Grassi et avec le soutien de Mario Apollonio et Virgilio Tosi, constitue le premier théâtre stable d'Italie, inaugurant un modèle de théâtre public qui se répand ensuite dans la péninsule. Le Piccolo Teatro entend défendre, dans le moment de reconstruction de l'après-guerre, un théâtre qui révèle la communauté à elle-même et qui participe à la fondation d'une société et d'un homme nouveaux, tranchant avec la période fasciste. Strehler et Grassi, respectivement artiste directeur et administrateur, développent ce projet d'un théâtre pour tous jusqu'au moment des contestations de 1968. L'esthétique de Strehler et le fonctionnement du théâtre stable sont alors profondément remis en cause par la jeune génération, le mouvement des coopératives et la scène alternative. Strehler quitte la direction du Piccolo, laissant Grassi seul à la tête du théâtre. En 1972, Grassi étant devenu surintendant de la Scala, Strehler reprend la direction du théâtre et, jusqu'à sa mort en décembre 1997, privilégie un théâtre qui se replie sur l'artisanat de la scène et interroge sa capacité à survivre dans une société où l'idée de communauté s'estompe et où le lien entre le théâtre et le monde s'avère délicat à tisser.

2. Giorgio Strehler, Appunti di regia Arlecchino 1984, Note su Arlecchino pubblicate nel programma di sala della stagione 1983-84, consultable en ligne sur: http://archivio.piccoloteatro.org/eurolab/ index.php?tipo $=6 \& \mathrm{ID}=64 \& \mathrm{imm}=1 \&$ contatore $=2 \&$ real $=0$ 
3. Renato Simoni (1875-1952), dramaturge et critique, a conduit à Milan, au début du XXème siècle, un travail autour de Carlo Gozzi et de la Commedia dell'arte mais sans utiliser de véritables masques, d'après le témoignage de Strehler.

4. Max Reinhardt, sous l'influence de Meyerhold, avait réabordé la Commedia dell'arte, en en reprenant l'esprit. Il met en scène le Serviteur de deux maitres en 1926 au festival de Salzbourg.

5. Strehler, Les Voies de la Création théâtrale, p. 141.

6. Giorgio Strehler, Appunti di regia Arlecchino tournée 1954 : riflessioni sullo spettacolo pubblicate sul programma di sala in occasione della tournée in America Latina del 1954, consultable en ligne sur: http://archivio.piccoloteatro.org/eurolab/index.php?tipo=6\&ID=59\&imm=1\&contatore $=0$ \&real $=0$ 7. L'expression est de Paolo Grassi. Elle désigne l'ensemble des nouvelles méthodes employées par l'administrateur et destinées à faire connaître, auprès de la population milanaise et italienne, le Piccolo Teatro et plus généralement l'art théâtral. Elle joue de la connotation religieuse du terme prosélytisme, qui peut renvoyer à l'influence de la religion et de la Démocratie chrétienne au sein de la société italienne.

8. La première version est celle du 24 juillet 1947, représentée au Piccolo Teatro, avec des décors de Gianni Ratto, des costumes d'Ebe Colciaghi et des musiques de Fiorenzo Carpi. Distribution : Armando Alzelmo, Antonio Battistella, Anna Maestri, Marcello Moretti, Franco Parenti, Dino Riefolo, Gianni Santuccio, Roberto Villa, Otello Zago, Elena Zareschi. La seconde version est celle du 17 avril 1952, avec des décors de Gianni Ratto, des costumes d'Ebe Colciaghi, des musiques de Fiorenzo Carpi et des masques d'Amleto Sartori. Distribution: Antonio Battistella, Marcello Bertini, Giulio Bosetti, Nino Cestari, Raoul Grassilli, Vittoria Martello, Marcello Moretti, Franco Parenti, Checco Rissone, Adriana Sivieri, Lia Zoppelli. Théâtre Quirino (Rome). La troisième version est donnée le 27 août 1956, avec des décors et des costumes d'Ezio Frigerio, des musiques de Fiorenzo Carpi et des masques d'Amleto Sartori. [distribution]: Lidia Alfonsi, Antonio Battistella, Marcello Bertini, Narcisa Bonati, Marina Bonfigli, Tino Carraro, Giulio Chazalettes, Raoul Consonni, Angelo Corti, Ottavio Fanfani, Valentina Fortunato, Franco Graziosi, Ezio Marano, Gianfranco Mauri, Marcello Moretti, Franco Parenti, Antonio Pierfederici, Relda Ridoni, Checco Rissone. Royal Lyceum Theatre (Festival d'Edimbourg). La quatrième version est représentée le 24 juin 1973 avec des décors et des costumes d'Ezio Frigerio, des musiques de Fiorenzo Carpi et des masques d'Amleto et de Donato Sartori. Distribution : Cip Barcellini, Ginella Bertacchi, Angelo Corti, Giancarlo Dettori, Franco Graziosi, Andrea Matteuzzi, Gianfranco Mauri, Marisa Minelli, Giorgio Naddi, Anna Saia, Ferruccio Soleri, Gianrico Tedeschi. Villa Comunale Milano. La cinquième version dite édition de l'Odéon 1977 est créée le 4 octobre 1977 avec des décors et des costumes d'Ezio Frigerio, des musiques de Fiorenzo Carpi et des masques d'Amleto et de Donato Sartori. Distribution : Armando Benetti, Carlo Boso, Roberto Chevalier, Piergiorgio Fasolo, Franco Graziosi, Susanna Marcomeni, Gianfranco Mauri, Marisa Minelli, Nico Pepe, Anna Saia, Ferruccio Soleri, Enzo Tarascio, Elio Veller, Pamela Villoresi. Théâtre de l'Odéon, Paris. La sixième version, dite de l'Adieu voit le jour 14 mai 1987 avec des décors d'Ezio Frigerio, des costumes de Franca Squarciapino, des musiques de Fiorenzo Carpi et des masques d'Amleto et de Donato Sartori. Distribution: Walter Battagliola, Anania Battagliola, Narcisa Bonati, Enrico Bonavera, Roberto Chevalier, Ettore Conti, Giancarlo Dettori, Anna Ferraresi, Ettore Gaipa, Valerio Geroldi, Franco Graziosi, Andrea Jonasson, Giulia Lazzarini, Sergio Lomazzi, Riccardo Magherini, Susanna Marcomeni, Gianfranco Mauri, Marisa Minelli, Edmondo Sannazaro, Ferruccio Soleri, Enzo Tarascio, Mario Valdemarin. Piccolo Teatro de Milan. La septième édition, dite du Bonjour est présentée le 30 octobre 1990 avec des décors d'Ezio Frigerio, des costumes de Luisa Spinatelli, des musiques de Fiorenzo Carpi et des masques d'Amleto Sartori et de Natale Panaro. Distribution : Giovani I, Ferruccio Soleri. Piccolo Teatro de Milan.

9. Les emplois dramatiques de la Commedia dell'arte sont d'une part les personnages masqués c'est-à dire les zanni (serviteurs) et les vieux ridicules (par exemple Pantalon) qui fonctionnent par couples et d'autre part les amoureux, personnages sérieux et non masqués. 
10. Le sonnet de l'édition de 1753 n'était pas toujours pris en compte dans les représentations de la pièce, qui se terminait alors sur une demande de pardon d'Arlequin à ses maîtres. La traduction utilisée ici est celle de Valeria Tasca : Arlequin serviteur de deux maîtres, ed.bilingue, GF Flammarion, Paris, 2005, p. 286.

11. Giorgio Strehler, Ancora e sempre "Arlecchino", Appunti di regia Arlecchino 1984 Note su Arlecchino pubblicate nel programma di sala della stagione 1983-84, op.cit.

12. Giorgio Strehler, L'Arlecchino dell'Addio, Appunti di regia Arlecchino 1987, Riflessioni pubblicate sul programma di sala di Arlecchino servitore di due padroni per le celebrazioni del $40^{\circ}$ anniversario della fondazione del Piccolo Teatro ; consultable en ligne sur : http://archivio.piccoloteatro.org/eurolab/ index.php?tipo $=6 \& \mathrm{ID}=62 \& \mathrm{imm}=1 \&$ contatore $=3 \&$ real $=0$

13. Ibidem.

14. L'allusion renvoie aux mises en scène strehlériennes de La Tempête (La Tempesta) de W. Shakespeare en 1978 et de L'Orage (Temporale) d'A. Strindberg en 1980.

15. Ferrucio Soleri, in Soleri, servitore di un padrone : Arlecchino, L'Arena, 29 mai 1987.

16. Giorgio Strehler, Buongiorno "Arlecchino"! Appunti sulla cosiddetta Edizione del "Buongiorno" messa in scena nel 1990 come saggio di diploma degli allievi del "Corso Copeau" della Scuola di Teatro, consultable en ligne sur: http://archivio.piccoloteatro.org/eurolab/index.php? tipo $=6 \& \mathrm{ID}=63 \& \mathrm{imm}=1 \&$ contatore $=4 \&$ real $=0$

17. Expression de Jacques Copeau, in Jacques Copeau, Souvenirs du Vieux Colombier, Nouvelles éditions latines, 1931, p. 83.

18. Giorgio Strehler, Buongiorno "Arlecchino"! Appunti sulla cosiddetta Edizione del "Buongiorno" messa in scena nel 1990 come saggio di diploma degli allievi del "Corso Copeau" della Scuola di Teatro, op. cit.

19. Giorgio Strehler, Ancora e sempre "Arlecchino", Note su Arlecchino pubblicate nel programma di sala della stagione 1983-84, Appunti di regia Arlecchino 1984, op.cit.

20. Ibidem.

21. Giorgio Strehler reprend essentiellement ses mises en scène de Brecht et de Goldoni. Il donnera quatre éditions de La Bonne Ame du Se-Tchouan, trois de L'Opéra de Quat'sous et de La Trilogie de la Villégiature; deux de Barouf à Chioggia et du Campiello. En dehors de ces auteurs, il reprendra deux fois La Cerisaie de Tchékhov, La Tempête et le montage shakespearien du Jeu des puissants et cinq fois les Géants de la Montagne de Pirandello.

22. Giorgio Strehler, Per un teatro umano. Pensieri scritti, parlati e attuati, Feltrinelli, Milano, 1974, pp. 94-95.

\section{RÉSUMÉS}

Giorgio Strehler, au cours de ses cinquante années à la tête du Piccolo Teatro de Milan, a mis sept fois en scène, chaque fois de manière différente, Arlequin, serviteur de deux maitres de Carlo Goldoni. Cette reprise, d'une durée inédite dans l'histoire du théâtre, s'est poursuivie après sa mort en 1997, L'Arlequin étant toujours à l'affiche du Piccolo Teatro en 2013. Cette persévérance dans un geste de reprise conduit à en interroger les raisons et à en déplier les variations. La reprise de l'Arlequin permet de renouer avec l'héritage de la Commedia dell'arte dans un geste de réappropriation qui dépasse la reconstitution pour mener une recherche sur l'essence du jeu et 
sur l'évolution d'un geste artistique au fil des ans qui provoque la métamorphose de la reprise et la confronte aux enjeux de la mémoire, de l'invention scénique et de la programmation.

INDEX

Mots-clés : Strehler (Giorgio), Arlequin, Piccolo Teatro, Goldoni, Commedia dell'arte, reconstitution, réappropriation, transmission

\section{AUTEUR}

\section{AUDE ASTIER}

Doctorante en études théâtrales à l'Université Paris X et à l'ENS de Lyon, membre fondateur de la revue Agôn et agrégée de lettres modernes, Aude Astier enseigne actuellement à l'Université d'Artois en tant qu'ATER en Arts du spectacle. 